\title{
Protecting the brain against bad news
}

\author{
Cite as: CMAJ 2021 March 22;193:E428-9. doi: 10.1503/cmaj.1095928
}

Posted on cmajnews.com on March 5, 2021

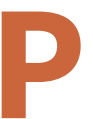

eople are consuming more news, and more negative news, during the coronavirus disease 2019 pandemic, which may be contributing to worsening mental health. But some psychologists say cultivating optimism can help enhance the brain's defences against bad news.

The impulse to consume negative news can be difficult to resist. "We are evolutionarily wired to screen for and anticipate danger, which is why keeping our fingers on the pulse of bad news may trick us into feeling more prepared," says Cecille Ahrens, clinical director of Transcend Therapy in San Diego, California. Following the news may be necessary to stay informed, especially during a crisis. But Ahrens says the feelings of fear, sadness and anger triggered by negative headlines can keep people stuck in a "pattern of frequent monitoring," leading to worse mood and more anxious scrolling.

This negative spiral - lately dubbed "doomscrolling" - can take a toll on mental health. Studies have linked the consumption of bad news to increased distress, anxiety and depression, even when the news in question is relatively mundane. According to Graham Davey, professor emeritus of psychology at the University of Sussex, exposure to bad news can make personal worries seem worse and even cause "acute stress reactions and some symptoms of posttraumatic stress disorder that can be quite long-lasting."

Normally, the brain's inferior frontal gyrus (IFG) selectively filters bad news when updating beliefs based on new information. This optimistic bias is the reason why people tend to assume they will live longer and suffer fewer accidents than their peers. A stronger optimistic

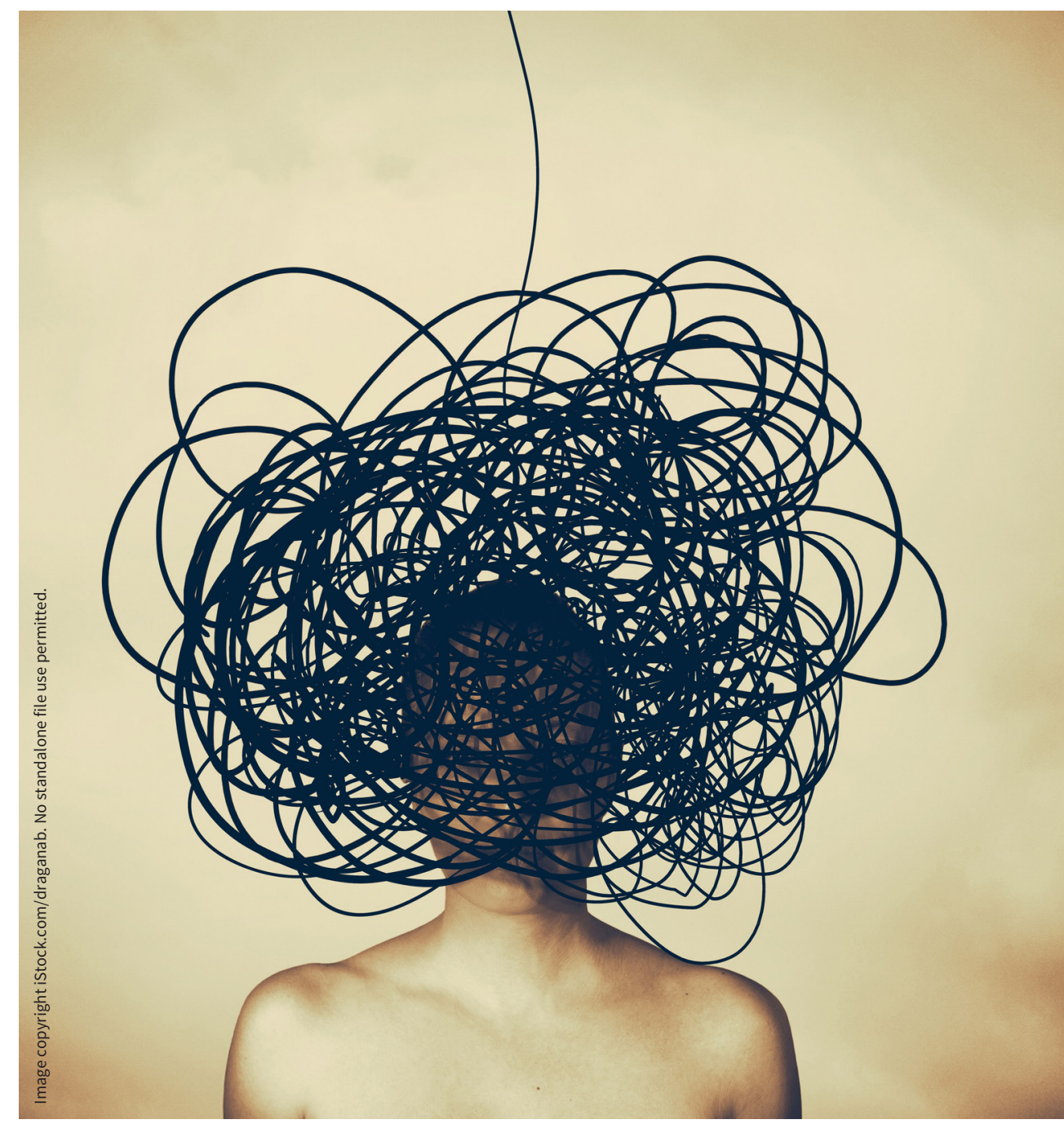

Training the brain to look on the bright side may counter doomscrolling.

bias also appears to confer health benefits. Optimists enjoy better physical and mental health, and are more resilient, even though they assess risk less accurately than pessimists.

Scientists at the University of London have temporarily converted optimists into pessimists by suppressing IFG activity. And others have found that the brain's bad news filter shuts off under threat - a feature that's helpful in lifeor-death situations but self-defeating when triggered by doomscrolling.

But what about strengthening this filter? Some researchers contend that boosting optimism may help people cope better with bad news without unplugging entirely. 
One cross-cultural study found that optimists, people with high levels of general trust, and those who do not believe in conspiracy theories experienced less fear than pessimists when consuming information about the pandemic and were more likely to follow public health measures.

Another recent study found that optimism protected older people from obsessively searching for medical information online and from the negative psychological effects of that behaviour. According to the authors, "given this benefit of optimism during the current pandemic, it is essential to consider ways in which it might be enhanced." For example, "previous studies suggested that engaging in social activities, religious involvement, social support, physical activities, or practising gratitude might enhance optimism and coping."

Studies show that positive psychology interventions, such as gratitude journaling and imagining a "best future self," increase optimism. In one 2017 trial, patients with heart disease who received optimism training reported sustained improvements in optimism, hope, life satisfaction, and anxiety two and four months later. In other cases, the effects of these interventions appear to be short-lived.

Ahrens says positive psychology interventions aim to train the brain to attend to positive experiences so that they "leave a deeper mark on our psyche and our bodies" over time. American psychologist Tara Brach refers to this process as "turning a state into a trait."

However, critics of positive psychology science have raised concerns about its replicability, reliance on self-report measures, and ties with religious groups. Even so, the approach remains popular in North America, with Yale University and the United States military launching positive psychology programs.

Some psychologists have noted that these programs are not one-size-fits-all. According to Julie Norem of Wellesley College, positive psychology interventions may backfire for people who cope with anxiety by preparing defensively for worst-case scenarios. "It's hard for them to force themselves to think positively, and it doesn't address the real issue of their anxiety."

In such cases, Davey recommends selective news engagement. "Watch the news only at specific times of day and make sure you have some activities available after consuming news that will lift your mood, like listening to upbeat music, exercise of some kind, or a good soak in a relaxing, warm bath."

Robin Blades, Berkeley, California, with files from Lauren Vogel

Content licence: This is an Open Access article distributed in accordance with the terms of the Creative Commons Attribution (CC BY-NC-ND 4.0) licence, which permits use, distribution and reproduction in any medium, provided that the original publication is properly cited, the use is noncommercial (i.e., research or educational use), and no modifications or adaptations are made. See: https://creativecommons.org/ licenses/by-nc-nd/4.0/ 